\title{
Reducing community infection by institutional interventions: a minimal SEIRJ model applied to COVID-19
}

Emmanuel Lance Christopher VI Plan ( $\nabla$ emmanuellcvmedilloplan@duytan.edu.vn )

Duy Tan University https://orcid.org/0000-0003-2268-424X

Minh Tan Man

Duy Tan University https://orcid.org/0000-0002-4318-4482

Binh Dinh

Duy Tan University

Thuy Bui

Duy Tan University

Manh Le

Duy Tan University

Article

Keywords: Coronavirus, SEIRJ model, infection rate

Posted Date: August 26th, 2020

DOI: https://doi.org/10.21203/rs.3.rs-56644/v1

License: (a) (i) This work is licensed under a Creative Commons Attribution 4.0 International License. Read Full License 


\title{
Reducing community infection by institutional interventions: a minimal SEIRJ model applied to COVID-19
}

\author{
Emmanuel Lance Christopher VI M. Plan ${ }^{1,2, *}$, Minh Tan Man ${ }^{1,2}$, Thanh Binh Dinh ${ }^{1,2}$, \\ Phuong Thuy Bui ${ }^{1,3}$, and Duy Manh Le $e^{1,3, * *}$
}

\author{
${ }^{1}$ Institute of Theoretical and Applied Research, Duy Tan University, Hanoi, 100 000, Vietnam \\ ${ }^{2}$ Faculty of Natural Sciences, Duy Tan University, Danang, 550 000, Viet Nam \\ ${ }^{3}$ Faculty of Pharmacy, Duy Tan University, Danang, 550 000, Vietnam \\ *emmanuellcvmedilloplan@duytan.edu.vn \\ **leduymanh@duytan.edu.vn
}

\begin{abstract}
The 2019 Coronavirus Disease continues to spread, and in the interim absence of a proven cure and vaccine, preventive community interventions continue to be implemented across countries. Here we propose and analyze a minimal compartmental model that simultaneously incorporates three such interventions: lockdowns, rapid isolation, and institutional propaganda. Using a modified SEIRJ model, we vary three different factors in the quantity that determines the effective rate of infection. Estimates of the model parameters are obtained from publicly-available data and were used to simulate various combinations of interventions. Our results show that a combination of interventions could decrease the total number of infected individuals by one or two orders of magnitude and keep the number of active cases low. In particular we show that lockdowns need not be very stringent if proper social distancing via media awareness and rapid identification and isolation is practiced. We also highlight that an effective isolation system strongly improves the effect of these interventions.
\end{abstract}

\section{Introduction}

As of writing, there are almost 20 million confirmed cases of the 2019 Coronavirus Disease (COVID-19) worldwide arising from several waves of infection in different parts of the globe. Since there has yet to be a cure against the viral disease as of writing and the vaccine candidates still require a few more months before completing the clinical trials, one main direction of the campaign against this disease is geared towards how medical and governmental institutions can prevent its transmission so as to minimize the number of confirmed cases and to not overwhelm the healthcare system.

Governments have long implemented community-wide interventions, such as contact tracing, social distancing, and lockdowns. Contact tracing involves tracking down and isolating individuals suspected of being in contact with infected persons and have high probability of being infectious. This is necessary for COVID-19 because of its long incubation period and the possibility of asymptomatic or presymptomatic transmission ${ }^{1-8}$. Social distancing, in this case the required two-meter gap between two individuals, prevents an individual's immediate contact to aerosols from an infected individual. While this may be difficult to fully implement, government mandate coupled with increased media mileage brings awareness to the community. Models have shown that increased consciousness or explicitly minimizing contacts reduce disease transmission ${ }^{9-14}$. While social distancing is normally implemented in models via closures of establishments ${ }^{12,13}$ and thus akin to a lockdown approach, strategies based on a network model have shown how selective social distancing delays and flattens the infection curve by limiting interaction and disease transmission into within small groups ${ }^{14}$. Lastly, lockdowns involve controlled or complete isolation of a local area. Lockdowns have been shown to be effective in controlling the spread of diseases ${ }^{11-13,15-18}$; of note is that the subexponential growth of the number of infections in China after the initial spread is attributed to lockdown measures ${ }^{15}$. Most countries implemented lockdowns under varying versions, stages and duration, but such lockdowns incur unsustainable economic and social costs.

The measures above have been incorporated into mathematical models that aim to capture disease transmission. One widely-used model are the deterministic compartmental models which can be used to track and predict the number of infected individuals. Fractions of the population are categorized into compartments according to their health status, and these values vary over time depending on the transition rates between compartments. The adaptability and versatility of compartmental models to model population demographics and interventions put the model as among the first tools of any epidemiologist.

Compartmental models that account for lockdowns and isolation of suspected and confirmed cases have been used, for 
example to model SARS in 2003, among others ${ }^{11,12,19,20}$. In these models, healthy individuals categorized as Susceptibles (S) become infected at a rate of $\beta I S$, where $I$ is the proportion of the population that is infected (I) and $S$ the proportion in $\mathrm{S}$. Lockdowns, quarantine and isolation are normally included using more compartments and thus alter the form of transmission rate. Although several works have presented results notably on the lockdown and isolation interventions, to our knowledge, a study that includes the effect of media and perception on the transmission rate and therefore vary all three factors of the term $\beta I S$ has yet to be done.

To focus on the interventions and their effect, we propose a minimal compartmental model that can evaluate the relative effect of these strategies and quantify their effectiveness in order to reduce both the cumulative number of infected people and the actual number of infected people at any time. Using our modified SEIRJ model, we show that five compartments is sufficient to simulate the effect of the interventions while keeping track of the total number of actively-infected cases. These compartments are the susceptibles S, exposed E (infected but not infectious), infectious I (undiagnosed, presymptomatic, asymptomatic, or mildly symptomatic), removed or recovered $\mathrm{R}$ (including deaths), and isolated $\mathrm{J}$ (confirmed and currently active). The proportion of the population that belongs to each compartment is denoted in its italicized form (e.g. $S$ for $\mathrm{S}$ ). The transition rates from one compartment into another, evident in the schematic provided in figure 1, are described in the Methods section.

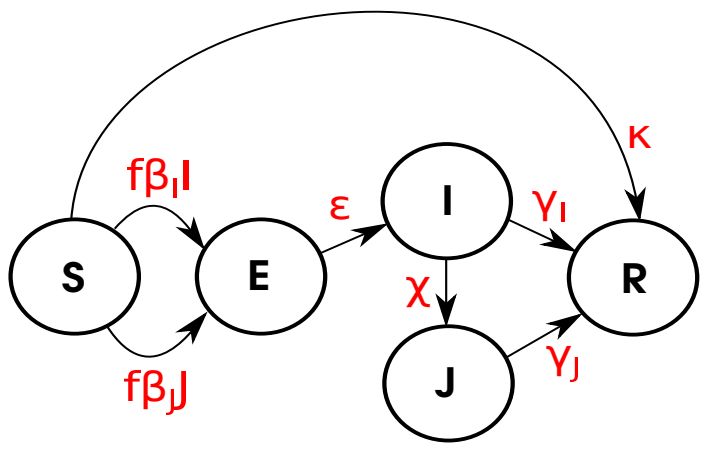

Figure 1. A schematic of the SEIRJ-with-containment model used in this work.

Lockdowns reduce the fraction $S$ by isolating individuals from $\mathrm{S}$ into $\mathrm{R}$; this is controlled by the parameter $\kappa$. Contact tracing and quick isolation (via $\chi$ ) work by reducing the proportion $I$ that infects those in $\mathrm{S}$ at the rate of $\beta_{I}$ and moving them into $\mathrm{J}$ whose transmission rate $\beta_{J}$ is ideally lower. Lastly, the effect of institutional propaganda on the spread of a disease is seen by coupling the transmission rates $\beta_{I}, \beta_{J}$ to a media function $0 \leq f \leq 1^{9,21}$, which captures how responsive the population is to government and media pronouncements. In particular, $f$ is lower when the awareness of the population is increased. The specific form of $f$ that we adopt in this work is $f(C(t) ; p)=1-\frac{C}{C+p}$, where $C(t)$ is the total number of confirmed cases in a country and $p$ is a parameter which measures the country's sensitivty to media (see Methods for more details).

We first provide results of fitting existing data into the model so as to give sense to the model parameters. The practical and epidemiological questions will then be addressed by simulating the model under the obtained parameter estimates for different different degrees of intervention. In particular, we will estimate the total number of confirmed cases $C$ and the number of infected people $I+J$ at any time since medical and societal decisions such as implementing lockdowns, managing manpower, and purchasing medical supplies require these values. The possibility of implementing weaker lockdowns is addressed by assessing when the number of infected individuals reach a particular threshold. Lastly, we also show how increased protection inside isolation facilities $\left(\beta_{J}=0\right)$ coupled with rapid isolation strongly translates to lower number of transmissions.

\section{Results}

\section{Parameter estimation}

The parameter estimates obtained for select countries are presented in table 1. Graphs of the fitting are provided in the supplementary material (Fig. S1). Most parameter values lie within a reasonable range, although the reproductive numbers may be bigger than reported in other studies.

The countries with minimal cases in number or as a fraction of their population are the same countries indicating relatively strong containment measures. For instance, Taiwan yielded $\kappa \approx 0.09$ and New Zealand has $\kappa \approx 0.06$. South Korea and Sweden exhibited strong containment as well even though they have not enforced nationwide lockdowns - the model nevertheless felt containment effects, which means that people stayed at home. The western European countries Italy, France, and Germany have $\kappa \approx 0.017$, which could be used as a benchmark for a moderately strong lockdown, as may be perceived in the news. 
Lastly, the estimates for Mexico and Spain reveal that the effect of any lockdown during this period did not translate to effective containment.

Another characteristic of the countries with minimal cases is a very low value of $10^{-4}<p<10^{-3}$, which means that the population responded to the reported number of confirmed cases by social distancing. Germany, Hubei, and Spain, also have very strong community response. In contrast, Brazil, Chile, and Peru have $p>10^{-2}$. This translates to, after a period of 90 days, a reduction of only $1-f(C(90) ; p) \approx 22-39 \%$ of their transmission rates $\beta_{I}$ or $\beta_{J}$, whereas most other countries have reduced theirs by more than $50 \%$ (see last column of table 1 ). Other countries with less than $50 \%$ reduction in the transmission rates are Taiwan, New Zealand, and Mexico.

Lastly, the countries that performed rapid isolation (mean of $\chi^{-1}$ around 3 days or less) are Italy, Mexico, South Korea, and Sweden. The two latter countries are known to not have implemented strict lockdown measures and instead focused more on contact tracing and identification of possible cases. These allowed rapid isolation and reduced an infected individual's transmission coefficient from $\beta_{I}$ to $\beta_{J}$.

We remark that the numbers for Chile reflected slow $\left(\chi^{-1}>14\right)$ and ineffective isolation $\left(\beta_{I} \approx \beta_{J}\right)$. This provides another point to be interpreted. Effective isolation $\left(\beta_{J} \approx 0\right)$ is another trait exhibited by New Zealand and Taiwan (also Hubei). As will be reinforced in the simulations below, effective isolation can strongly reduce transmission.

\section{Epidemiological characteristics}

\section{Reproductive number and number of confirmed cases}

The basic reproductive number $R_{0}$ is defined as the average number of secondary infected individuals from a primary infected person over the course of the infectiousness period in the absence of any other intervention, and so is evaluated at time $t=0^{22}$. An $R_{0}>1$ indicates an uncontrolled spread of the disease while an $R_{0}$ below unity indicates that the disease will not spread. In our model, $R_{0}$ incorporates effects the effect of media, although this is negligible because $C(0) \approx 0$ at time $t=0$. The $R_{0}$ in this case, calculated via the next generation matrix ${ }^{22,23}$, is given by

$$
R_{0}=\frac{\left(f \beta_{I} \gamma_{J}+f \beta_{J} \chi\right)}{\gamma_{J}\left(\gamma_{I}+\chi\right)} .
$$

The value of $R_{0}$ given the representative exploratory parameters (corresponding to those of Italy in table 1) are shown in the black curve (with $\times$ marks) in figure 2 as a function of the isolation rate $\chi$. Here, the values of $R_{0}$ are larger than those in the

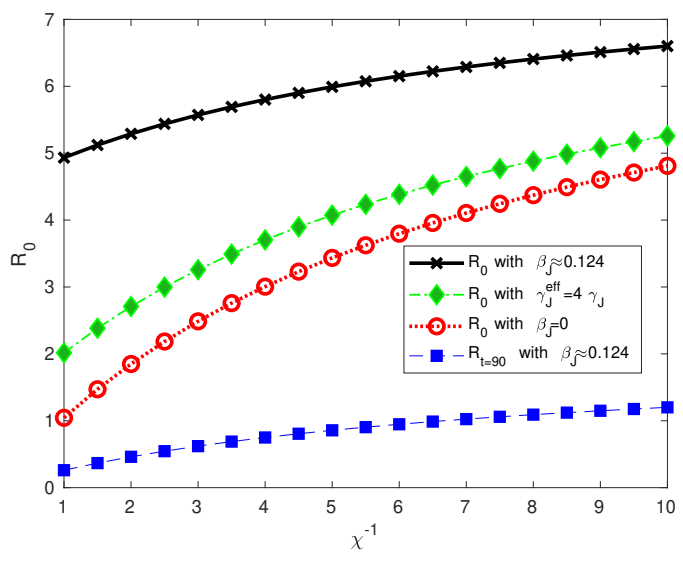

Figure 2. The reproductive number given by Eq. 1 calculated on the day $(t=0)$ when $J>100$. The black curve (marked by $\times$, straight line) gives the curve for $\beta_{J} \approx 0.124$. The green gurve (marked with $\downarrow$, dot-dashed line) uses the same parameters except with a removal rate $\gamma_{J} 4$ times as given. The red curve (marked by $\circ$, dotted line) gives the curve with $\beta_{J}=0.0$. The blue curve (marked by $\mathbf{\square}$ ) gives the reproductive number integrating the effect of media by day 90.

literature largely because our model fits a recovery rate with a mean hospitalization time of around 30 days, whereas the actual time that a patient may be infectious may be much less. If the patient remains infectious in the hospital at an average of around 9 days $\left(\gamma_{J} \approx 0.11\right), R_{0}$ can be much lower (green curve, diamond markers). Alternatively, well-protected medical facilities will result into no hospital-acquired infection $\left(\beta_{J}=0\right)$, also resulting in a lower $R_{0}$ (red curve, open circles). In any of the cases above, rapid identification and isolation of infected cases reduce transmissibility. However, the reproductive number remains well above 1, implying that the disease still spreads. 
The presence of interventions can drive down the effective reproductive number. For instance, we can account for the effect of the media function $f$ via the incorporation of the number of confirmed individuals, thereby reducing the effective transmission coefficients $\beta_{I}$. If calculated ninety days after the initial spread (hence a larger value of $C$ ), we obtain $R_{0}<1$ given that $\chi^{-1}<6.5$ (blue curve in the figure). Moreover, if we include the time-dependent effect of the containment parameter $\kappa$ and estimate a time-dependent reproductive number $R_{t}=S(t) \cdot R_{0}$ as the product of the basic reproductive number and the remaining number of susceptible persons at that time, the reproductive number $R_{t}$ may start above 1 at $t=0$, but drops below 1 after a period of around 30-80 days, depending on the degree of the intervention (see Fig. S2 of the supplementary material).

The reproductive number is but an indication of the spread; practical decision depends on the number of infected pesons. Figure 3 shows the total number of infected individuals for different values of the parameters $\kappa, \chi^{-1}$, and $p$. For Italy

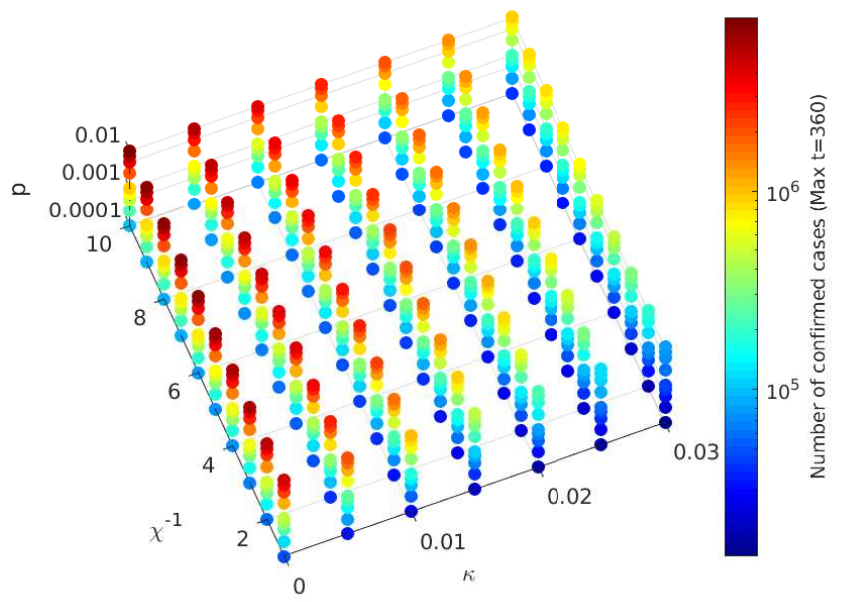

Figure 3. The total number of confirmed individuals $C=\int \chi I \mathrm{~d} t$ as a function of the intervention parameters. The simulation assumes the population of Italy $\left.\left(N_{\text {pop }}=6.055 \times 10^{7}\right)\right)$.

$\left(N_{\text {pop }}=6.055 \times 10^{7}\right)$, the number of confirmed cases was about $2 \times 10^{5}$ on May $22(t=90)$, and is fitted with $\kappa \approx 0.0169$ and $\chi^{-1} \approx 3$. Visually, the effect of enforcing lockdowns, rapid isolation, and an effective media, can be seen clearly in the color gradient in all directions. There are less cases if containment is stronger (higher $\kappa$ ), isolation is more rapid (lower $\chi^{-1}$ ), and media awareness is stronger (smaller $p$ ). Figure 4 quantifies the results from three dimensions into two dimensions for the parameter set $\kappa=0.01, \chi=1 / 5, p=0.001$. A decrease of one order of magnitude in the cumulative number of infected
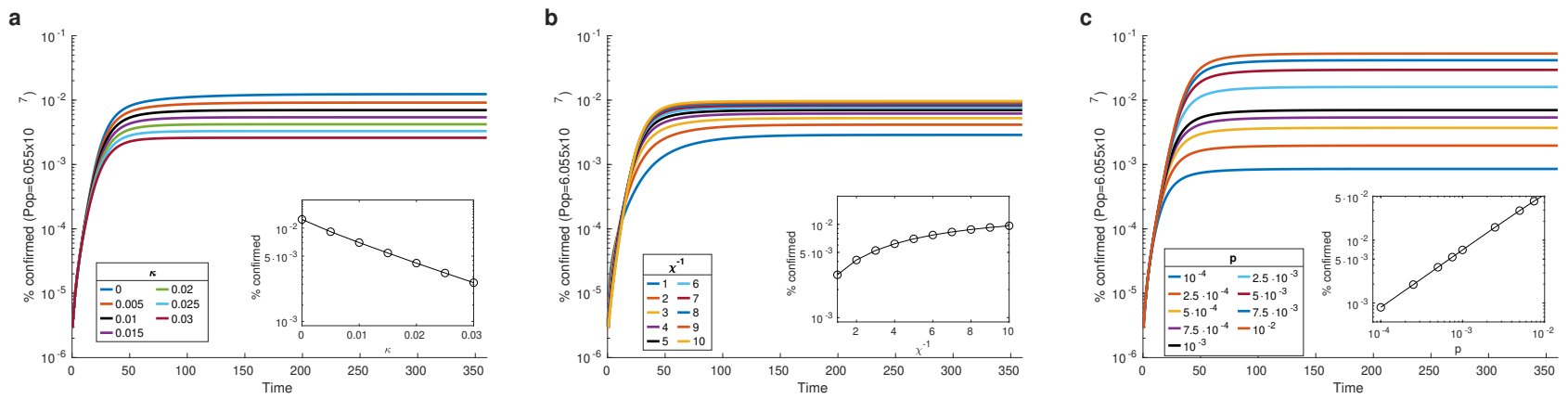

Figure 4. The effect of varying control parameters (a) $\kappa$, (b) $\chi^{-1}$, and (c) $p$ on the total number of confirmed cases over time. The black curve in each figure corresponds to the parameter set $\kappa=0.01, \chi=1 / 5, p=0.001$. The insets show the maximum number of confirmed as a function of the variables.

persons can be observed as the containment parameter $\kappa$ increases from 0.0 to 0.03 . There is likewise a steady decrease in the number of infected persons as the mean time to isolation decreases. Lastly, the effect of media can spell a decrease of two orders of magnitude in the number of cases from countries where media or government propaganda is ignored to one where the community is strongly cooperative. These interventions, individually or in conjunction of another, clearly minimize the spread of the disease. 


\section{Peaking}

Given that $R_{0}>1$ and spreading was inevitable, the next important question is whether or not healthcare facilities can cope with the number of persons requiring medical care at any point in time. Governments aimed to flatten the infectious curve and delay the peak in order to prepare for sufficient medical facilities for the influx of patients. Our model can provide estimates on the number of infected individuals given the various interventions explored and estimate when the peak may occur; estimates of healthcare capacity is beyond the model and the scope of this study.

A full three-dimensional figure showing the peak times and the peak number of infections is provided in the supplementary material (Fig. S3). Here the base parameter set $\kappa=0.01, \chi=1 / 5, p=0.001$ is explored in two dimensions to provide a clearer picture (figure 5). In all cases of our simulation, the number of infected individuals decrease after peaking two to three months
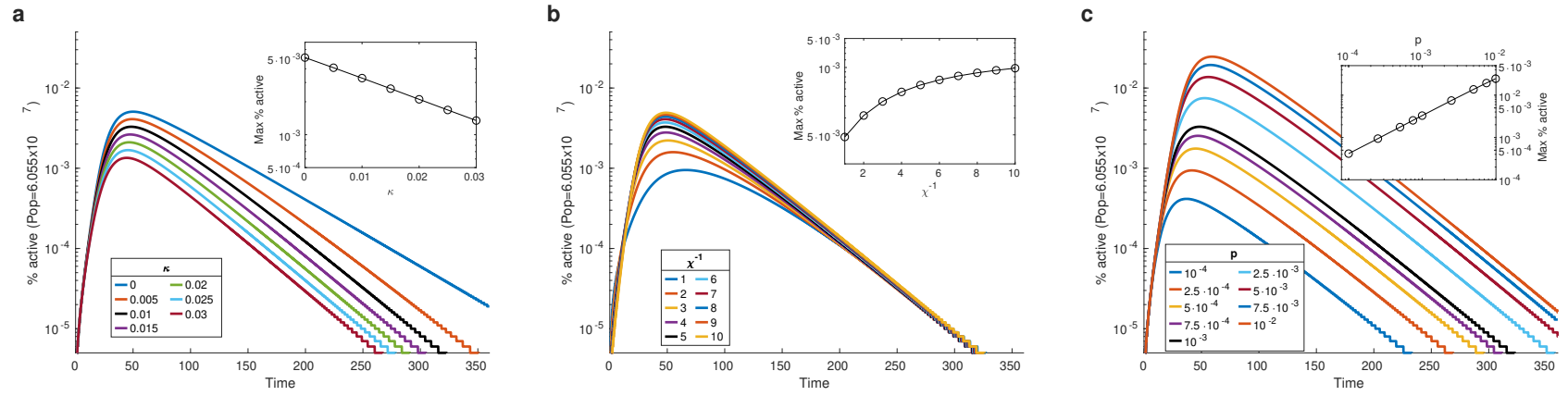

Figure 5. The number of active cases over time, varying the parameters $(a) \kappa,(b) \chi^{-1}$, and (c) $p$. The legend for this follows from Fig. 4 . The isets show the number of active cases at peak.

from the start of the epidemic. The time to peak is weakly affected by containment; there is however a sizable decrease in the peak number of cases. A stronger awareness imparted via media decreases the value of the peaks (by one or two orders of magnitude) but the peak also occurs earlier. Lastly, delaying the peak can be achieved by minimizing the mean infectious time of patients via quick isolation. The delay in the peak of course assumes that the probability that they can infect someone decreases during isolation, as is assumed in this study (in the case of Italy, $\beta_{J} \approx \beta_{I} / 10 \approx 0.12$ ). We remark that in one study conducted in China during the early stages of the spread of this disease, the isolated patients remained highly infectious ${ }^{3}$ with $\beta_{J} \approx 0.5 \beta_{I}$, possibly caused by a premature understanding of the disease.

\section{Lifting lockdowns and effective isolation}

In a completely mathematical language, we can estimate the amount of time needed to completely remove the virus. This time is achieved when $E+I+J<1 / N_{\text {pop }}$. The parameter set for Italy provides a dire situation where it is nearly impossible to remove the virus within a year (no figure shown, similar to figure 6a but mostly in dark red); the model would then have to be improved to account for possible seasonal effects.

A more promising scenario can be obtained if a contagion is loosely-defined to be controlled if the numbers are low and the infected patients are clearly identified. For instance, it is possible to estimate critical community size below which an epidemic is not expected to sustain itself ${ }^{24}$. If we relax the conditions by setting defining a "controlled" situation with $E+I+J<50 / N_{\text {pop }}$ and $\beta_{J}=0$, we obtain figure 6 a, which shows us the time to limit the disease to 50 individuals. While
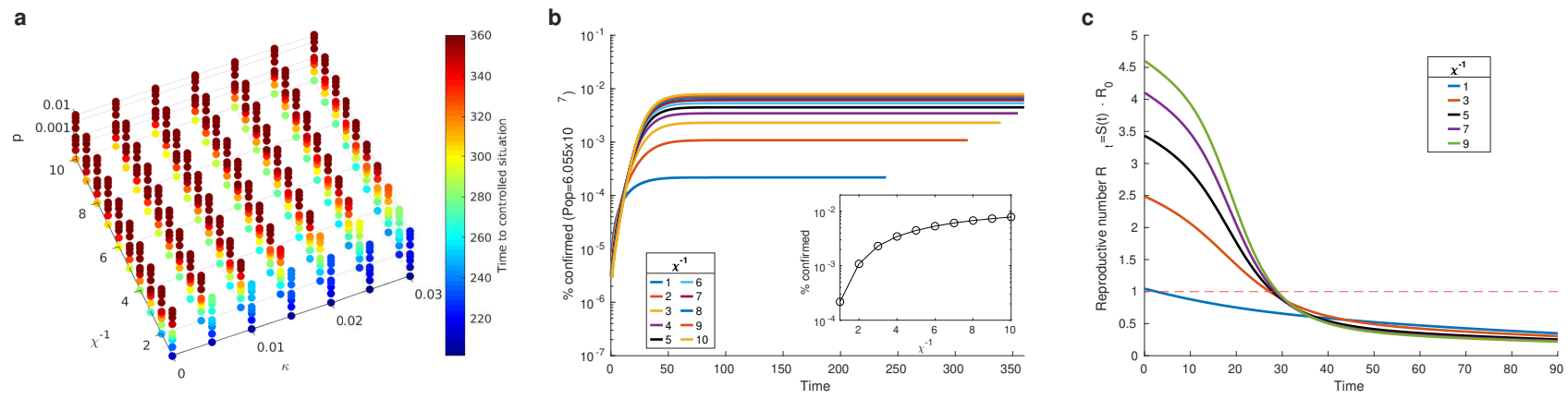

Figure 6. (a) Time to reach a controlled situation (less than 50 infected individuals) for the case $\beta_{J}=0$. (b) Time series of the number of confirmed cases for different values of $\chi^{-1}$ for the case $\beta_{J}=0$. (c) Time-varying reproductive number $R_{t}$ for the case $\beta_{J}=0$. 
most of these simulations still require at least 300 days to achieve a controlled situation, note that some degree of containment, rapid isolation (less than 4 days), and strong media awareness can bring the situation under control in 6 to 8 months. At time of writing, Italy still has around $10^{4}$ active cases 5 months into this pandemic, and so may indeed take a few more months to reduce this number to a few tens. We also emphasize that the effect of rapid isolation is more pronounced for the ideal case of no symptomatic transmission $\left(\beta_{J}=0\right)$, as shown in figure $6 \mathrm{~b}$ (compare with figure $4 \mathrm{~b}$ ). Also, the time-dependent reproductive number $R_{t}$ starts low especially for cases when isolation occurs fast enough (figure $6 \mathrm{c}$ ).

Our simulations show that assuming a certain degree of alternative interventions and a good awareness of the community, lockdowns need not be very strict and still achieve a controllable situation. For instance, using the parameters of Italy (note: $\beta_{J} \neq 0$ ), a weaker lockdown $\kappa=0.005$ but very strong awareness $p=0.0001$ (similar to that exhibited by South Korea) and modestly quick isolation $\chi=1 / 3$ could have resulted in lower number of total or active cases, as seen in the blue curves in figure 7. Had Italy been more well-informed about COVID-19 at that time, the number of cases could have been at least $75 \%$

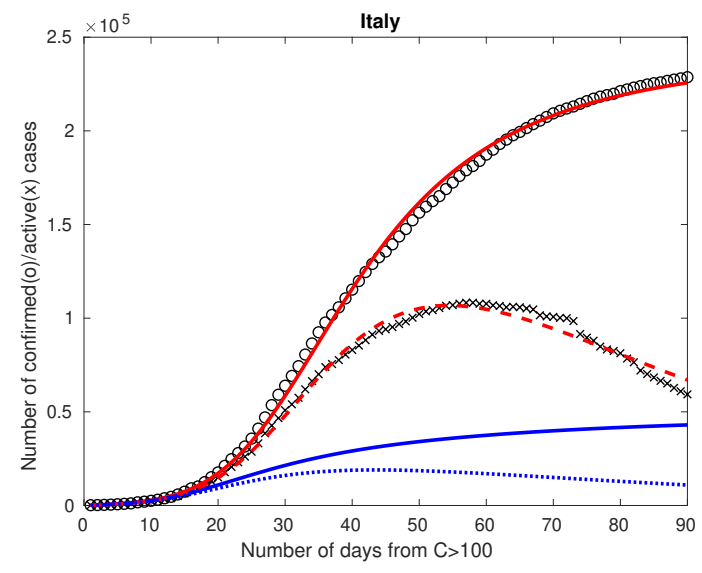

Figure 7. The epidemic curves for Italy with day 1 corresponding to when $C>100$. The markers $\circ$ and $\times$ give the number of confirmed and active cases respectively; the red curves are the fitted values. The blue curves give the estimated number of confirmed (solid) and active (dotted) cases for the same parameters except $\kappa=0.005, \chi=1 / 3, p=0.0001$.

lower. Such a limited spread of infection further results in a shorter duration of the disease. Using the parameters as used to obtain the blue curve, 100 stochastic realizations of the model result in the disease being limited to 50 individuals after a mean of 325 days (minimum: 283 days, maximum: 365 days, standard deviation: 21 days).

\section{Discussion}

In this report we have proposed the minimal SEIRJ model that captures three different interventions: increasing social consciousness of the population captured via a time-dependent media function, implementing containment policies of the government, and the rapid isolation of individuals.

The effect of lockdown and rapid isolation in decreasing the number of infected individuals support existing literature, while our study shows that increased social awareness could reduce the number of confirmed cases even if containment is not stringent and isolation rates are moderate. We also present how different combinations of these interventions could limit the total or actual number of infected individuals. Moreover, we note that in the absence of vaccines, medicines and seasonal effects on the virus, the disease is expected to stay in the population, unless people exposed to the virus are well-isolated. We highlight that an effective isolation strongly increases the effect of these interventions.

This work thus recommends lockdowns (if there are no economic and social repercussions) or a relaxed version if coupled with extremely strong community awareness and rapid isolation via massive testing and effective contact tracing, as well as proper medical isolation facilities and equipment. A deterministic model such as ours guarantee that easing lockdowns is safe if $R_{t}<1$ once the lockdown is lifted. If the quarantined population return to the susceptible state, and under the assumption that the infectiousness and removal rates remain the same, $R_{t}$ can easily increase above 1 and a second wave of infection is probable. The estimate when to lift lockdowns can be improved and is better addressed by using stochastic models which can give interval estimates of when the community is disease-free $9,14,18,24,25$. While we have implemented a stochastic realization of the model to obtain the time to control the situation, this requires time and computational resources. Further studies must be done and account for varied dynamics. For instance, agent-based models ${ }^{18}$ and social network models ${ }^{14}$ have also been used to address similar community interventions. 


\section{Methods}

\section{Theoretical background}

The following system (2) of differential equations is the modified SEIRJ model that accounts for the containment, isolation and the general awareness of the community. A schematic of this model is shown in figure 1, where each compartment is shown inside a circle and the transition rates from any two linked compartments are labeled in red.

$$
\begin{aligned}
d S & =-f \beta_{I} I S-f \beta_{J} J S-\kappa S \\
d E & =-\varepsilon E+f \beta_{I} I S+f \beta_{J} J S \\
d I & =\varepsilon E-\left(\gamma_{I}+\chi\right) I \\
d R & =\kappa S+\gamma_{I} I+\gamma_{J} J \\
d J & =\chi I-\gamma_{J} J .
\end{aligned}
$$

The cumulative number of confirmed patients is given by $C=\int_{t} \chi I \mathrm{~d} t$, i.e. the entrants in $\mathrm{J}$ but without recovery. In our model all values are rescaled into fractions of the total population $N_{\text {pop }}$ so that $N=S+E+I+R+J=1$.

A fraction of the population in S gets infected via asymptomatic transmission at the rate of $f \beta_{I} I(t)$, or symptomatic transmission at the rate of $f \beta_{J} J(t)$, possibly inside hospitals ${ }^{8}$. The virus incubates inside an exposed individual for a mean number of $\varepsilon^{-1}$ days. Once infectious, an individual can either recover at a rate of $\gamma_{I}$, or be a confirmed case and isolated after an average of $\chi^{-1}$ days. The removal rate during isolation is given by $\gamma_{J}$ and can be substantially long (reports show that recovery occur with a median of 20 days $^{26}$, some even reaching 37 days). Note that death is implicitly included in the removed compartment R. Thus $\gamma_{J}$ is better understood as a removal rate rather than a recovery rate from the active case compartment $\mathrm{J}$.

Containment or quarantine measures enforced by the government appear in the transition from the susceptible compartment $\mathrm{S}$ into $\mathrm{R}$ at a rate of $\kappa$. In the absence of infections, the differential equation $d S=-\kappa S$ has the solution $S(t)=\exp (-\kappa t)$. An intuitive understanding of the containment rate then is that it takes $\kappa^{-1}$ days to effectively quarantine $e^{-1} \approx 36.79 \%$ of the population. Note that in reality lockdowns are implemented immediately and may be represented by a step-function ${ }^{17}$. Nevertheless, substantial mobility remains particularly for persons involved in essential production, healthcare, and governance. Moreover, the virus may still spread within households, in hospitals, and in shops. Several modelling studies have supported the effectiveness of lockdowns ${ }^{11,15-18}$.

Isolation and case identification is enforced by healthcare personnel at a rate of $\chi$, which is the inverse of the mean time from the onset of being infectious until diagnosis. Previous studies of COVID-19 show that individuals may be infectious hours or days before symptom onset ${ }^{13,27,28}$. Moreover, proper isolation is not always practiced because individuals may find it difficult to interpret mild symptoms and diagnosis, especially in the absence of known contact with COVID-19 cases, occurs days after symptom onset. We remark that effective isolation increases the controllability of an outbreak as shown by a stochastic branching-type agent-based model in spite of having reproductive numbers above the critical value ${ }^{25}$.

The effect of media and government propaganda on social awareness is included in the model as a function $f$ that reduces the transmission rates $\beta_{I}$ and $\beta_{J}$ as a function of the number of reported cases $C$ and a parameter $p>0$ that measures the responsiveness or awareness of the community. Various non-monotonic incidence rates $f \beta I$ are available in literature ${ }^{9,21}$; here we adopt the function $f(C(t) ; p)=1-\frac{C}{C+p}=(1+C / p)^{-1}$, which is a decreasing function of the number of confirmed cases $C \cdot N_{p o p}$. A higher value of $p$ approaches the case where people are less affected by the media pronouncements on the number of confirmed cases, whereas a lower value of $p$ allows the term $C / p$ to majorize the denominator, resulting to a fraction $f(C(t) ; p)<1$, reducing the transmission rate.

\section{Parameter estimation}

Time-series data of the daily cumulative number of confirmed, dead, and recovered individuals from all countries starting on January 22, 2020, until July 30, 2020 were obtained from the John Hopkins University Center for Systems Science and Engineering database ${ }^{29}$. Countries that have a large enough number of infected individuals, that exhibited no second waves of infection in the time duration, and for which their COVID-19 responses are known in mainstream media are selected. The time series for both confirmed $\left(N_{p o p} \cdot C\right)$ and active cases $\left(N_{\text {pop }} \cdot J\right)$ were then fitted starting from the date when there are at least 100 confirmed individuals $\left(N_{p o p} \cdot C>100\right)$ over a duration of 90 days. This parameter estimation provides a simple validation of the model and more importantly, a qualitative interpretation of the resulting values of the parameters. The parameter fits were obtained using the trust-region algorithm for nonlinear least squares in Matlab. The initial parameter estimates for the fitting $\left(\beta_{I}=0.8, \beta_{J}=\beta_{I} / 10, \varepsilon=0.2, \gamma_{I}=0.15, \gamma_{J}=0.05, p=0.001, \kappa=0.01, \chi=0.2\right)$ are based on existing literature. Reference values of these parameters are provided in the supplementary material (Table S1). 


\section{Numerical integration}

The parameter results of Italy were then chosen as representative values for the parameters to be used in the simulation, and the model is evaluated for different parameter values of the interventions $\left(\kappa, \chi^{-1}\right.$, and $\left.p\right)$. The differential equations (2) were solved using 4th-order Runge-Kutta method, and the initial data used is based on Italy. The total population $N_{\text {pop }} \approx 6.055 \times 10^{7}$ is based on the 2019 population, with an assumption that the number of confirmed cases is $C=J=155 / N_{p o p}$, and the number of asymptomatic cases $I=2 J$ is assumed to be 2 times the number of confirmed cases, which is within the range of previous fitting simulations and surveyed conditions ${ }^{5-7,15}$. The estimated serial intervals ${ }^{7,27,30}$ as well as incubation times (see Table S1 of the supplementary material) are both around 5-7 days, and so we find it reasonable that given $I+J$ actual infections, there are already $R_{0}=2.4$ times as many exposed individuals as $I+J$, where $R_{0}$ is the basic reproductive number; its value is obtained from literature rather than the simulation results. We assume that no case had yet been recovered. Note that since the media function $f$ is time-dependent, it is updated at every time step. The simulations are capped at $t=360$ (days) and is stopped if there are no more infected individuals: $N_{p o p}(E+I+J)<1$.

\section{Stochastic realization}

The deterministic system (2) can be simulated stochastically by using the Gillespie algorithm, which was initially conceived to provide a stochastic model for chemical reactions ${ }^{31}$. Adopted for infectious disease modelling, transitions from one compartment into another are considered as events and are assigned a random transition time. At any point in time, the time to the next event is taken as the minimum of all these transition times, and the corresponding event is implemented ${ }^{9,32}$. The mean of the distribution times are the inverses of the transition rates. Owing to computational restrictions, we simulate (2) with

$N_{\text {pop, rescale }}=3 \times 10^{6}$ and an initial $J \cdot N_{\text {pop }}=\operatorname{ceiling}\left(155 \cdot \frac{\mathrm{N}_{\text {pop,rescale }}}{\mathrm{N}_{\text {pop }}}\right)=8$, and other relations between the initial conditions as before. The average of 50 realizations using the parameters of Italy match the obtained fitting and the lie close to the data points (see figure $\mathrm{S} 4$ in the supplementary material). The simulation is terminated when $N_{p o p}(E+I+J)<50$ ("controlled" situation). Another simulation with modified interventions $(\kappa=0.005, \chi=1 / 3, p=0.0001)$ is performed and the maximum, minimum, and mean stopping time is reported.

\section{Data Availability}

The datasets used for the parameter-fitting are obtained from publicly available repository ${ }^{29}$.

\section{References}

1. Arons, M.M. et al. Presymptomatic SARS-CoV-2 Infections and Transmission in a Skilled Nursing Facility. New Eng. J. Med. 382, 2081-2090 (2020).

2. Gandhi, M., Yokoe, D. \& Havlir, D. Asymptomatic Transmission, the Achilles' Heel of Current Strategies to Control Covid-19. New Eng. J. Med. 382, 2158-2160 (2020).

3. Li, R. et al. Substantial undocumented infection facilitates the rapid dissemination of novel coronavirus (SARS-CoV-2). Science 368, 489-493 (2020).

4. Thompson, R., Lovell-Read, F. \& U. Obolski. Time from Symptom Onset to Hospitalisation of Coronavirus Disease 2019 (COVID-19) Cases: Implications for the Proportion of Transmissions from Infectors with Few Symptoms. J. Clin. Med. 9, 1297 (2020).

5. Gutdbjartsson, D.F. et al. Spread of SARS-CoV-2 in the Icelandic Population. N. Engl. J. Med. 382, 2302-2315 (2020).

6. Ganyani, T. et al. Estimating the generation interval for coronavirus disease (COVID-19) based on symptom onset data, March 2020. Eurosurveillance 25, 2000257 (2020).

7. Lavezzo, E. et al. Suppression of COVID-19 outbreak in the municipality of Vo, Italy. Nature (2020).

8. Basteiro, A. et al. Seroprevalence of antibodies against SARS-CoV-2 among health care workers in a large Spanish reference hospital. Nature Comm. 11, 3500 (2020).

9. Collinson, S. \& Heffernan, J. Modelling strategies for controlling SARS outbreaks. BMC Public Health 14, 376 (2014).

10. Goscé, L., Barton, D. \& A. Johansson. Analytical Modelling of the Spread of Disease in Confined and Crowded Spaces. Scientific Rep. 4, 4856 (2015).

11. Giordano, G. et al. Modelling the COVID-19 epidemic and implementation of population-wide interventions in Italy. Nature Med. 26 855-860 (2020).

12. B. Nussbaumer-Streit et al. Quarantine alone or in combination with other public health measures to control COVID-19: a rapid review. Cochrane Database of Systematic Reviews 4, CD013574 (2020). 
13. Ferguson, N. et al. Report 9: Impact of non-pharmaceutical interventions (NPIs) to reduce COVID-19 mortality and healthcare demand. Imperial College London MRC Centre for Global Infectious Disease Analysis 10.25561/77482 (2020).

14. Block, P. et al. Social network-based distancing strategies to flatten the COVID-19 curve in a post-lockdown world Nature Human Behaviour 4, 588-596 (2020).

15. Maier, B. \& Brockmann D. Effective containment explains subexponential growth in recent confirmed COVID-19 cases in China. Science 368, 742-746 (2020).

16. Leung, K., Wu, J.T., Liu, D. \& Leung, G.M. First-wave COVID-19 transmissibility and severity in China outside Hubei after control measures and second-wave scenario planning: a modelling impact assessment. Lancet 395, 1382-1393 (2020).

17. Lin, Q. et al. A conceptual model for the coronavirus disease 2019 (COVID-19) outbreak in Wuhan, China with individual reaction and governmental action. Int. J. Infectious Diseases 93, 211-216 (2020).

18. Hoertel, N. et al. Lockdown exit strategies and risk of a second epidemic peak: a stochastic agent-based model of SARS-CoV-2 epidemic in France. medRxiv 10.1101/2020.04.30.20086264 (2020).

19. Gumel, A.B. et al. Modelling strategies for controlling SARS outbreaks. Proc. Royal Soc. B 271, 2223-2232 (2004).

20. Hsieh, Y.-H. et al. Impact of quarantine on the 2003 SARS outbreak: A retrospective modeling study. J. Theo. Bio. 244, $729-736$ (2020).

21. Sun, C., Yang, W., Arino, J. \& Khan, K. Effect of media-induced social distancing on disease transmission in a two patch setting. Mathematical Biosciences 230, 87-95 (2011).

22. Heffernan, J., Smith, R. \& Wahl, L. Perspectives on the basic reproductive ratio. J. Royal Soc. Interface 2, 281-293 (2005).

23. Diekmann, O., Heesterbeek, J. \& Roberts, M. The construction of next-generation matrices for compartmental epidemic models. J. Royal Soc. Interface 7, 873-885 (2009).

24. Cullen, R., Korobeinikov, A. \& Walker, W. Seasonality and critical community size for infectious diseases. The ANZIAM Journal 44, 501-512 (2003).

25. Hellewell, J. et al. Feasibility of controlling COVID-19 outbreaks by isolation of cases and contacts. Lancet $\mathbf{8}$, E488-E496 (2020).

26. Zhou, F. et al. Clinical course and risk factors for mortality of adult inpatients with COVID-19 in Wuhan, China: a retrospective cohort study. Lancet 395, 1054-1062 (2020).

27. Peak, C.M. et al. Individual quarantine versus active monitoring of contacts for the mitigation of COVID-19: a modelling study. Lancet (2020).

28. Zhang, W. Estimating the presymptomatic transmission of COVID19 using incubation period and serial interval data. medRxiv 10.1101/2020.04.02.20051318v1 (2020).

29. COVID-19 Data Repository by the Center for Systems Science and Engineering (CSSE) at Johns Hopkins University. https://github.com/CSSEGISandData/COVID-19 (2020).

30. Bi, Q. et al. Epidemiology and transmission of COVID-19 in 391 cases and 1286 of their close contacts in Shenzhen, China: a retrospective cohort study. Lancet 8, 911-919 (2020).

31. Gillespie, D.T. A general method for numerically simulating the stochastic time evolution of coupled chemical reactions. J. Comput. Phys. 22, 403-434 (1976).

32. Kiss, I., Miller, J. \& Simon, P.L. Stochastic simulation of epidemics in Mathematics of Epidemics on Networks 381-413 (Springer International, 2017).

\section{Acknowledgements}

The authors would like to acknowledge Nguyen Cat Ho and Trinh Xuan Hoang for useful discussions.

\section{Author contributions statement}

E.L.C.M.P., M.M.T. and L.D.M. conceived the study and the model, E.L.C.M.P., D.T.B, P.T.B., and L.D.M. produced and analysed the results. E.L.C.M.P. and L.D.M. wrote the manuscript and all authors reviewed and approved it for publication. 


\section{Competing interests}

The authors declare no competing interests.

\section{Additional information}

\section{Supplementary information}

Supplementary information for this paper is available at XXX.

\section{Tables}

This table provides the results of the parameter estimation of the model for select countries. 


\begin{tabular}{|c|c|c|c|c|c|c|c|c|c|c|c|c|}
\hline Country & $N_{\text {pop }}$ & $C_{t=90}$ & $\beta_{I}$ & $\beta_{J}$ & $\varepsilon$ & $\gamma_{I}$ & $\gamma_{J}$ & $R_{0}$ & $p$ & $\kappa$ & $\chi$ & $1-f(C(90) ; p)$ \\
\hline Brazil & 211049527 & 772416 & 0.76476 & 0.09324 & 0.17075 & 0.15186 & 0.05114 & 3.2 & 0.01306 & 0.00951 & 0.21485 & 0.21890 \\
\hline Chile & 18952038 & 167355 & 0.39442 & 0.36973 & 0.16649 & 0.14963 & 0.09393 & 3.0 & 0.01411 & 0.00748 & 0.06763 & 0.38487 \\
\hline France & 65129728 & 183309 & 0.99837 & 0.06163 & 0.21884 & 0.10000 & 0.02169 & 5.2 & 0.00102 & 0.01765 & 0.20292 & 0.73444 \\
\hline Germany & 83517045 & 182922 & 1.09399 & 0.33265 & 0.23141 & 0.10397 & 0.06529 & 7.0 & 0.00027 & 0.01660 & 0.18631 & 0.88850 \\
\hline China (Hubei only) & 57110000 & 68128 & 1.37891 & 0.00205 & 0.24980 & 0.13304 & 0.04750 & 3.5 & 0.00051 & 0.03989 & 0.26586 & 0.69915 \\
\hline Italy & 60550075 & 228658 & 1.20344 & 0.12408 & 0.21799 & 0.14967 & 0.02777 & 5.6 & 0.00125 & 0.01695 & 0.31254 & 0.75088 \\
\hline Mexico & 127575529 & 150264 & 0.71770 & 0.06159 & 0.16154 & 0.15981 & 0.16725 & 1.7 & 0.00190 & 0.00128 & 0.32777 & 0.38316 \\
\hline New Zealand & 4783063 & 1509 & 0.53926 & 0.00032 & 0.18436 & 0.16807 & 0.06286 & 1.3 & 0.00076 & 0.06006 & 0.25299 & 0.29266 \\
\hline Peru & 32510453 & 229736 & 0.70506 & 0.06808 & 0.17913 & 0.15244 & 0.03517 & 3.0 & 0.01484 & 0.00889 & 0.22076 & 0.32257 \\
\hline South Korea & 51225308 & 11110 & 1.27962 & 0.06976 & 0.22642 & 0.14385 & 0.04266 & 3.7 & 0.00008 & 0.02882 & 0.35949 & 0.72888 \\
\hline Spain & 46736776 & 239228 & 1.24900 & 0.22435 & 0.24998 & 0.14388 & 0.03956 & 6.9 & 0.00043 & 0.00235 & 0.20877 & 0.92255 \\
\hline Sweden & 10036379 & 40803 & 0.80907 & 0.07430 & 0.16362 & 0.14786 & 0.01000 & 7.0 & 0.00347 & 0.00913 & 0.53360 & 0.53979 \\
\hline Taiwan & 23773876 & 445 & 0.07062 & 0.00050 & 0.13356 & 0.19609 & 0.03613 & 0.2 & 0.00032 & 0.09412 & 0.09409 & 0.05462 \\
\hline
\end{tabular}

Table 1. The parameter estimates obtained from the fitting process. The last column gives the percentage reduction in the transmission rate calculated based on the obtained value of $p$ and the number of confirmed individuals $C(t=90)$. 
Figures

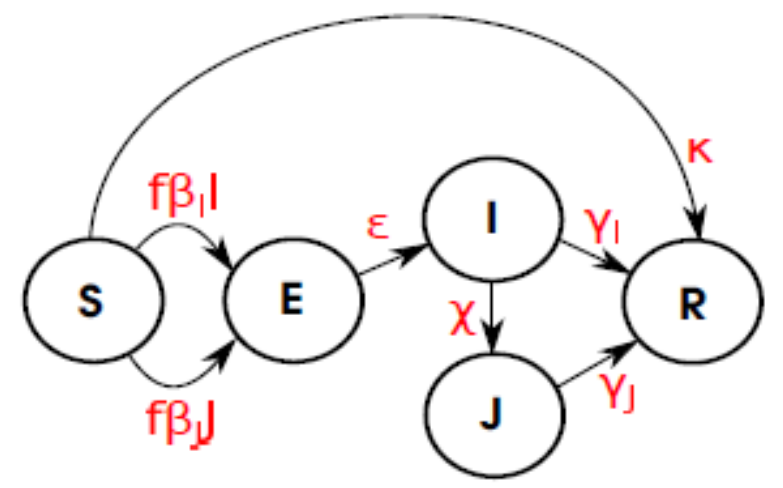

Figure 1

A schematic of the SEIRJ-with-containment model used in this work.

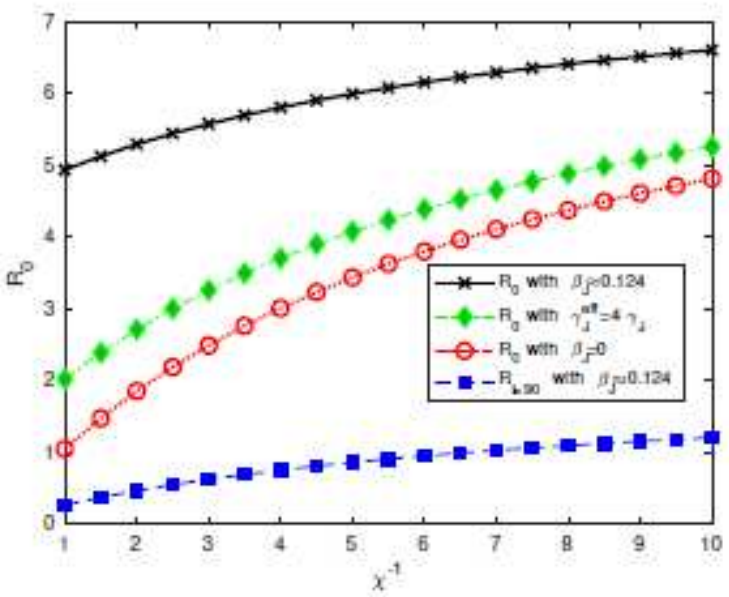

Figure 2

The reproductive number given by Eq. 1 


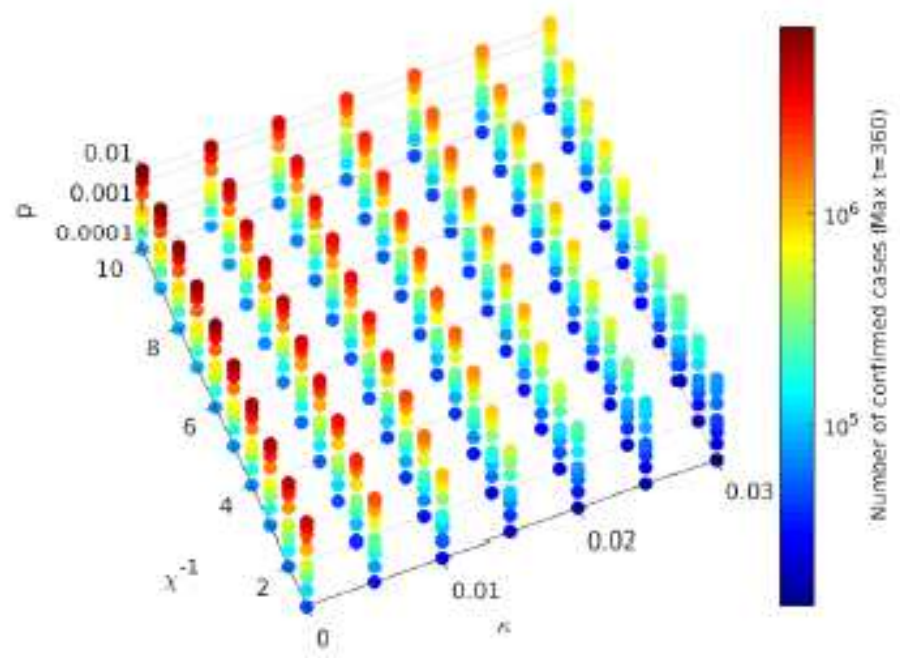

\section{Figure 3}

The total number of confirmed individuals

a

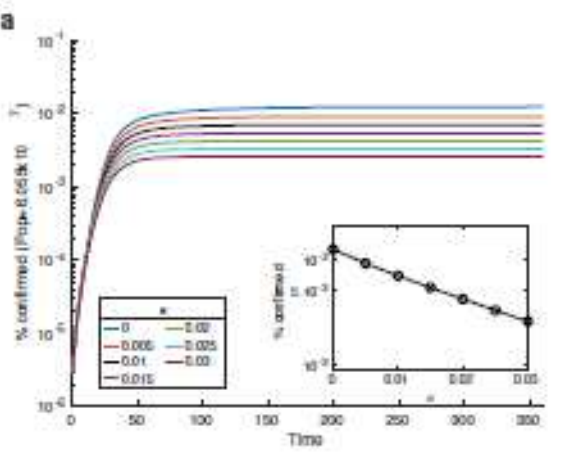

b

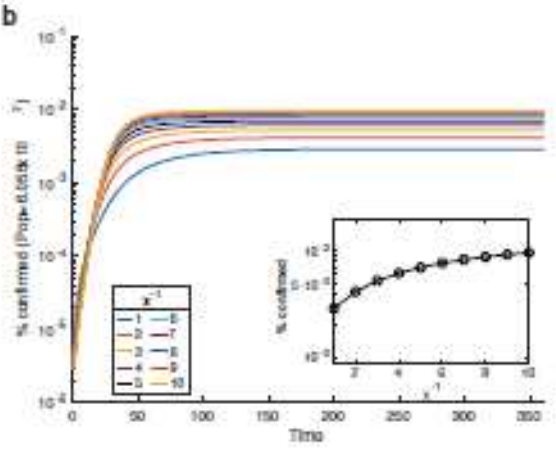

c

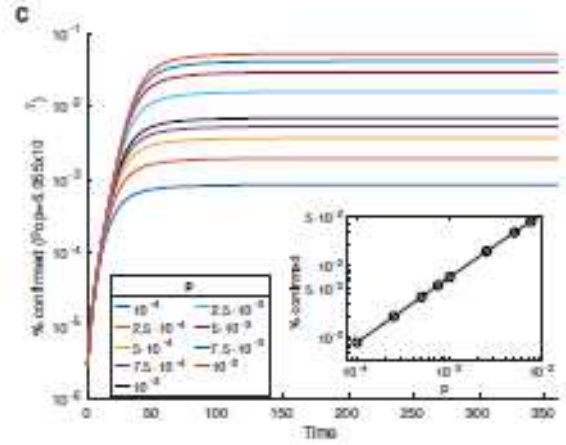

Figure 4

The effect of varying control parameters
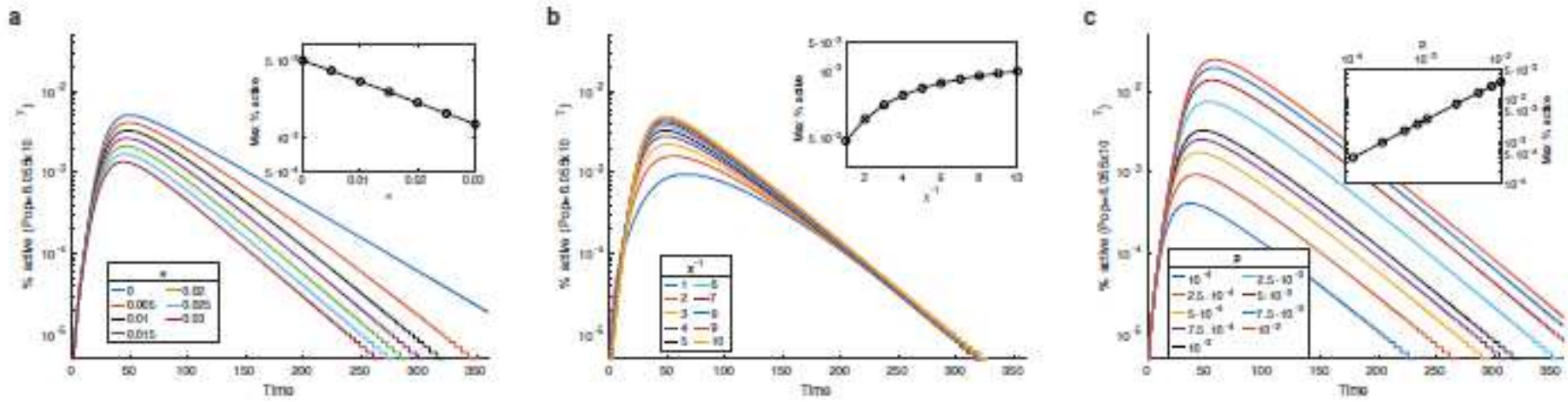

Figure 5

The number of active cases over time 
a

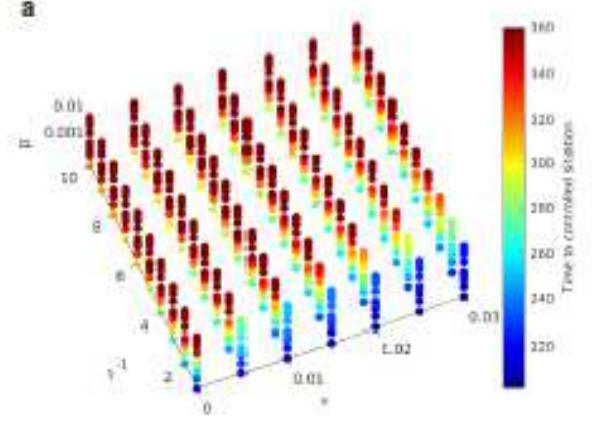

b

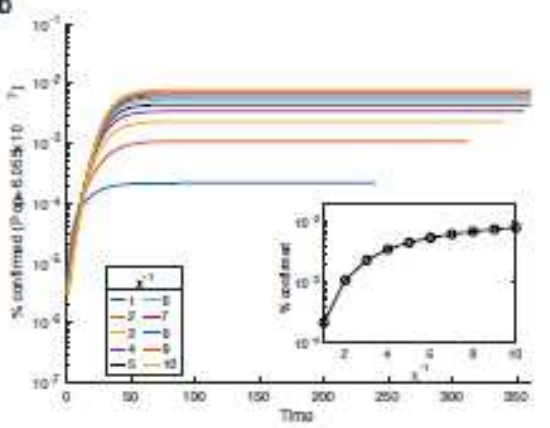

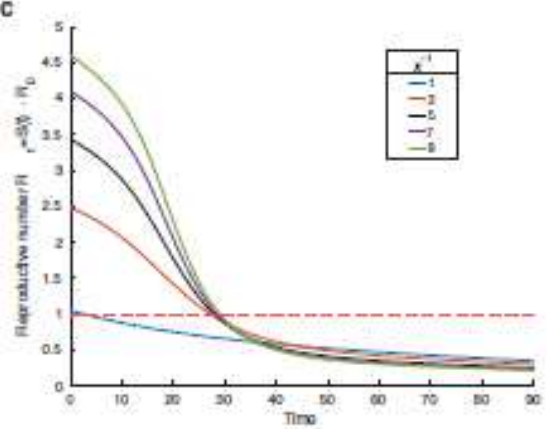

Figure 6

(a) Time to reach a controlled situation (less than 50 infected individuals) for the case bJ $=0$. (b) Time

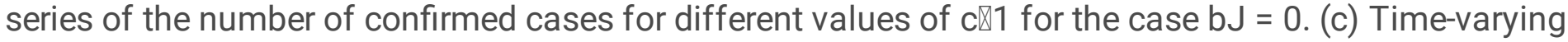
reproductive number $\mathrm{Rt}$ for the case $\mathrm{bJ}=0$.

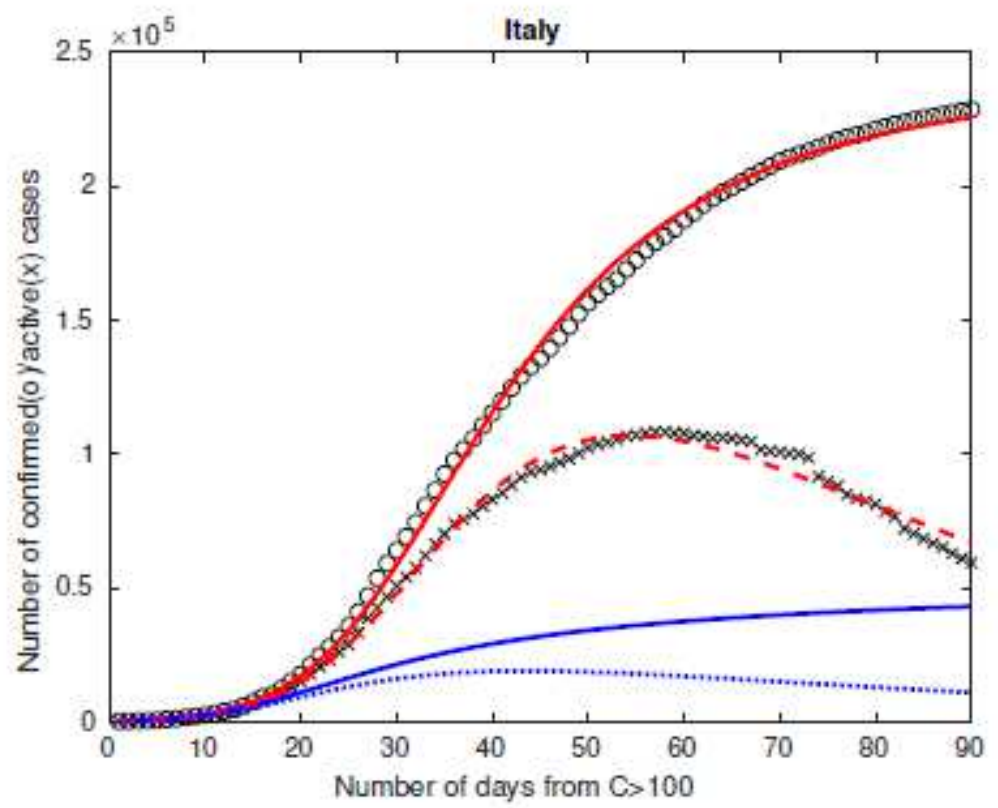

Figure 7

The epidemic curves for Italy

\section{Supplementary Files}

This is a list of supplementary files associated with this preprint. Click to download.

- suppsub01.pdf 